\title{
Left ventricular diastolic function in children measured by Doppler echocardiography: normal values and relation with growth
}

\author{
Frances A Bu'Lock, Martin G Mott, Robin P Martin
}

\begin{abstract}
Objectives-To determine normal values for variables of left ventricular diastolic function in children measured by Doppler echocardiography and their relations to body surface area (BSA).

Background-There is increasing interest in echocardiographic assessment of left ventricular diastolic function in children but normal data for children are limited.

Methods-Assessment of left ventricular diastolic function was performed in 130 normal participants (aged from 2.4 months to 19.6 years) from their transmitral flow patterns obtained by pulsed wave Doppler echocardiography.
\end{abstract}

Results-Centile charts for commonly used left ventricular diastolic functional variables plotted against BSA are presented. Peak early diastolic filling velocity and atrial phase filling velocity integral were independent of BSA. Although most other filling indices showed strong relations with BSA, some had more curvilinear relations with BSA due to additional interactions with heart rate. The increase in left ventricular filling with growth is largely achieved by an increase in the early "passive" contribution to filling. The slower heart rates of older children are associated with lower atrial phase filling velocities but increased filling time, so the atrial contribution to filling remains relatively constant.

Conclusions-Normal values of many left ventricular diastolic function variables change with growth in children and cannot be extrapolated from adult data. The data presented are suitable for use in size matched matched comparative studies of left ventricular function in children. Careful standardisation of echocardiographic protocols is necessary to ensure the validity of any comparisons.

Paediatric Cardiology and Oncology, Bristol Royal Hospital for Sick Children Sick Children, Bristol BS2 8BJ F A Bu'Lock

M G Mott

R P Martin

Correspondence to: Dr F A Bu'Lock, Department of Paediatric Cardiology, Alder Hey Children's Hospital, Eaton Road, Liverpool L12 2AP.

Accepted for publication

20 September 1994
The accurate assessment of ventricular function is becoming increasingly important in the management of children with heart disease, particularly with improvements in long-term survival for congenital and acquired heart disease.

High quality echocardiography equipment is now in general use. Echocardiography is readily performed and multiple examinations are rarely contraindicated. Measures of left ventricular systolic function obtained by $M$ mode echocardiography are widely accepted and used. Their utility, however, is limited in the presence of regional wall motion abnormalities or dyskinesia. ${ }^{1}$ It is also well recognised that symptomatic congestive cardiac failure may occur despite apparently normal cardiac function according to these indices in individuals with predominantly diastolic functional abnormalities. ${ }^{23}$ There is increasing interest in the use of Doppler echocardiography to measure transmitral flow velocity patterns as an index of left ventricular filling and hence diastolic function. This may provide a more global index of myocardial integrity. ${ }^{4-7}$

Normal values for diastolic filling parameters measured by Doppler echocardiography have been reported for preterm infants, ${ }^{8}$ neonates, ${ }^{9}$ and adults, ${ }^{6} 1011$ There are, however, few data for older infants, children, and adolescents, ${ }^{12}$ although these measurements are known to vary markedly with age and heart rate in adults. ${ }^{1314}$ There are no data on the relations of transmitral diastolic filling patterns to body surface area (BSA) in healthy children.

Normal values for diastolic left ventricular function in 130 children and adolescents under 20 years of age measured by Doppler echocardiography are presented here.

\section{Patients and methods}

Echocardiographic examination of 130 healthy children and adolescents from whom full clinical histories had been obtained and cardiovascular examinations performed was recorded. Height (m), weight $(\mathrm{kg})$, BSA $\left(\mathrm{m}^{2}\right)$ and systolic and diastolic blood pressures $(\mathrm{mm} \mathrm{Hg})$ were noted in each case (table 1$)$. There were 62 girls and 68 boys. Their ages ranged between 2.4 months and 19.6 (mean 7.52 ) years, with BSAs between 0.22 and 1.85 (mean 0.94 ) $\mathrm{m}^{2}$. No child had heart disease, as judged by history, clinical examination, and echocardiography, and all had normal systolic left ventricular function. Four had grade 1 or 2 ejection systolic murmurs diagnosed clinically as "innocent" and confirmed echocardiographically as having no pathological significance. 
Table 1 Cross correlation matrix of transmitral flow variables *

\begin{tabular}{|c|c|c|c|c|c|c|c|c|c|c|c|}
\hline & $E A c c$ & $E A c c T$ & $E D e c$ & $E D e c T$ & $A$ & $E V I$ & $A V I$ & $E A V I$ & $I V R T$ & $B S A$ & $H R$ \\
\hline E & 0.68 & 0 & 0.31 & 0.31 & 0.22 & 0.75 & 0.24 & 0.72 & -0.16 & 0 & -0.25 \\
\hline EAcc & - & -0.7 & 0.54 & -0.13 & 0.48 & 0.2 & 0.32 & $0 \cdot 2$ & -0.24 & -0.47 & 0.29 \\
\hline EAccT & - & - & -0.42 & 0.47 & -0.41 & 0.46 & -0.15 & 0.4 & 0.17 & 0.63 & -0.63 \\
\hline EDec & - & - & - & -0.74 & 0.41 & -0.27 & 0 & -0.25 & -0.5 & -0.57 & 0.48 \\
\hline EDecT & - & - & - & - & -0.2 & 0.8 & 0.16 & 0.76 & 0.43 & 0.62 & -0.63 \\
\hline A & - & - & - & - & - & 0 & 0.86 & $0 \cdot 16$ & -0.33 & -0.38 & 0.68 \\
\hline EVI & - & - & - & - & - & - & 0.2 & 0.94 & 0.15 & 0.45 & -0.63 \\
\hline AVI & - & - & - & - & - & - & - & 0.44 & -0.14 & 0 & 0.38 \\
\hline EAVI & - & - & - & - & - & - & - & - & 0.13 & 0.4 & -0.5 \\
\hline IVRT & - & - & - & - & - & - & - & - & - & 0.56 & -0.47 \\
\hline BSA & - & - & - & - & - & - & - & - & - & - & -0.64 \\
\hline
\end{tabular}

A, Peak atrial phase filling velocity $\left(\mathrm{ms}^{-1}\right)$; AVI, velocity integral of A wave $(\mathrm{cm}) ; \mathrm{BSA}$, body surface area $\left(\mathrm{m}^{2}\right) ; \mathrm{E}$, peak earl phase filling velocity $\left(\mathrm{ms}^{-1}\right)$; EA, EA ratio (E/A); EAcc, acceleration to peak E (ms ${ }^{-2}$ ); EAccT, time to peak E (ms); EAVI, velocity integral of entire transmitral flow complex $(\mathrm{cm})$; EDec, deceleration from peak E $\left(\mathrm{ms}^{-2}\right)$; EDecT, time from peak E to baseline (ms); EVI, velocity integral of E wave (cm); HR, heart rate (beats min $^{-1}$ ); IVRT, isovolumic relaxation time (ms); VIR, velocity integral ratio (EVI/EAVI). Table shows values of Pearson's correlation coefficient ( $r$ ) for relations between transmitral flow variables. $r$ Values in bold text denote those with $P<0.001$.

Full ethical approval was given for the study by the Bristol and Weston District Ethical Committee. Informed consent for each study was obtained from all parents and from the participants where appropriate.

\section{ECHOCARDIOGRAPHIC EXAMINATION}

Echocardiographic examination was performed in the left lateral semirecumbent position whenever possible. Sedation was not used and some small children were examined only in a semirecumbent position on their mother's lap. Examinations were performed by a single operator (FAB) using a Hewlett Packard 77020AC ultrasound scanner and recorded onto videotape. An electrocardiographic trace (ECG) and phonocardiogram were recorded simultaneously with the echocardiogram. Transducer frequency was $3.5 \mathrm{MHz}$ or $5 \mathrm{MHz}$, either or both being used for each patient, to provide optimal imaging and

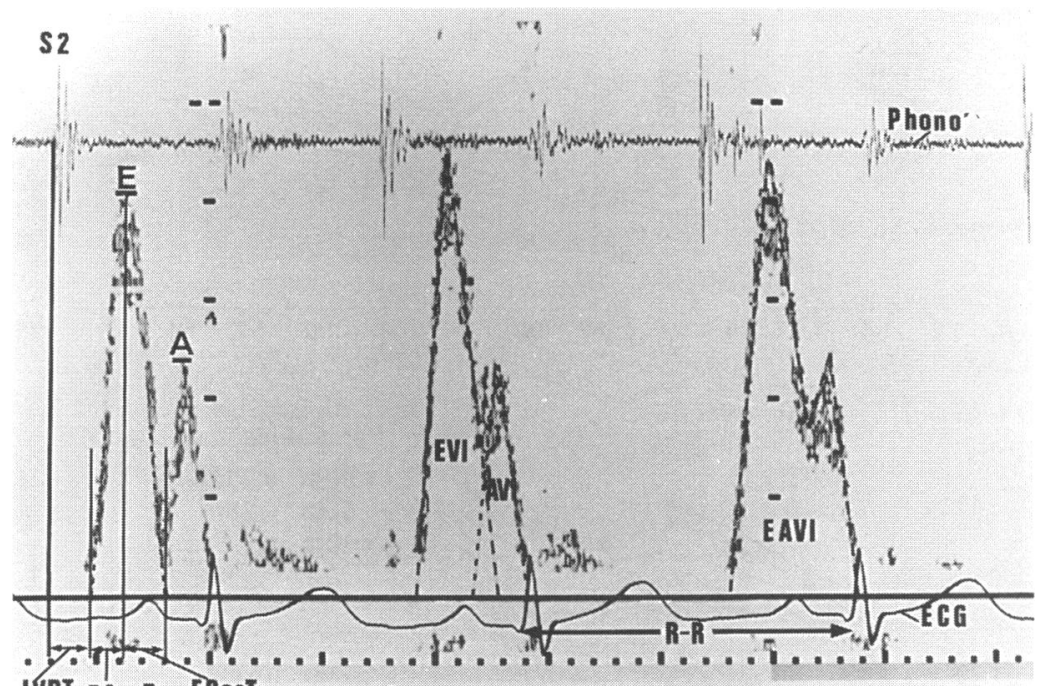

IVRT EACCT EDECT

Figure 1 Standard spectral Doppler trace of velocity against time obtained with the pulsed wave Doppler sample volume positioned between the tips of the mitral valve leaflets from the apical four chamber view. Chart speed is $100 \mathrm{~mm} / \mathrm{s}$, vertical velocity markers indicate $0.2 \mathrm{~ms}^{-1}$. Measurements are taken using the line of maximum spectral density. $A$ peak atrial phase filling velocity $\left(m s^{-1}\right) ; A V I$, velocity integral of $A$ wave $(\mathrm{cm}) ; E$, peak early phase filling velocity $\left(\mathrm{ms}^{-1}\right) ; E A c c T$, time to peak $E(\mathrm{~ms}) ; E A V I$, velocity integral of entire transmitral flow complex (cm); ECG, electrocardiographic trace; EDecT, time from peak $E$ to baseline (ms); EVI, velocity integral of E wave (cm); Phono',

phonocardiogram trace; $R R, R R$ interval (s); $S 2$, second heart sound; IVRT, isovolumic relaxation time (ms).
Doppler ultrasonographic recordings. "Chart speed" was $100 \mathrm{~mm} / \mathrm{s}$ for all recordings.

Transmitral flow velocity patterns were recorded from the apical four chamber view, with the smallest possible sample volume being positioned between the tips of the mitral valve leaflets. Participant and transducer position and angulation were adjusted as necessary to provide optimal alignment with transmitral flow and minimise the angle of incidence to left ventricular inflow; electronic angle correction was not used. Wall filter settings were kept to the minimum appropriate. Colour flow Doppler mapping was sometimes useful to clarify the spatial resolution of transmitral flow. A minimum of 30 cardiac cycles were recorded for each participant.

\section{ECHOCARDIOGRAPHIC ANALYSIS}

All scans were analysed by a single observer (FAB) from the previously recorded videotapes using the Hewlett Packard "on-line" analysis software. The mean of measurement from the five cardiac cycles with the highest peak early filling velocity $\left(E\left(\mathrm{~ms}^{-1}\right)\right)$ and a clear atrial phase filling peak $\left(\mathrm{A}\left(\mathrm{ms}^{-1}\right)\right)$ for each participant was used for analysis.

Peak $\mathrm{E}$ and $\mathrm{A}$ velocities were measured and the EA ratio (E/A) calculated (fig 1). Duration of acceleration to peak $E$ velocity and of deceleration from peak $\mathrm{E}$ velocity were measured in milliseconds using linear extrapolation of the velocity slopes through the wall filter to the baseline if necessary. Slope of the E velocity with time was recorded as the acceleration to and deceleration from (EDec) peak $\mathrm{E}$ velocity $\left(\mathrm{ms}^{-2}\right)$. The pressure half time (PHTE) (ms) of the deceleration from peak E velocity was measured using the formula:

$$
\text { PHTE }=(E-E / \sqrt{ } 2)(1 / E D e c)
$$

The isovolumic relaxation time (IVRT (ms)) was measured as the time interval between the first high frequency component of the second heart sound (recorded on the phonocardiogram) and the onset of transmitral flow. The "areas under the curves" of the E wave (EVI), A wave, and of the EA complex (EAVI) were measured in centimetres by planimetry of the line of maximum density. Where there was mid-diastolic flow distinct from the $\mathrm{E}$ and $\mathrm{A}$ waves it was included in the area under the 
Table 2 Normal percentile values for BSA independent variables

\begin{tabular}{llllll}
\hline \multicolumn{7}{c}{ Centiles } \\
\cline { 2 - 6 } & 10 & 25 & 50 & 75 & 90 \\
\hline Peak early phase filling velocity $\left(\mathrm{ms}^{-1}\right)$ & $0 \cdot 7$ & 0.76 & 0.84 & 0.91 & 0.97 \\
Velocity integral of A wave $(\mathrm{cm})$ & 2.34 & 2.82 & 3.35 & 3.81 & 4.36 \\
\hline
\end{tabular}

total flow curve, but not within either the $\mathrm{E}$ or A velocity integrals. The velocity integral ratio was calculated as EVI/EAVI. The RR interval (s) during the mitral recordings was also noted and heart rate (beats $\mathrm{min}^{-1}$ ) calculated as $60 / R R$

\section{Results}

STATISTICAL ANALYSIS

Univariate analyses confirmed that values of the study variables principally correlated with height, weight, BSA, age, heart rate, and systolic blood pressure. These variables highly correlated with each other and multivariate analysis was performed to establish the smallest possible set of predictor variables which described the variations in the study variables. Forward selection analysis showed that BSA (or a transformation of BSA) provided such a model for most study variables and that further refinement (and added complexity) through adjustment for heart rate was of little additional predictive value. For some variables, however, inherent curvilinearity in the data was noted. This finding was related to heart rate in a univariate model and was probably the result of the range of normal heart rates at any given BSA.

\section{RELATION TO GROWTH}

Peak $E$ velocity and $A$ velocity integral appeared largely independent of BSA; their overall centile range is given in table 2 .

There was also little change in diastolic blood pressure with BSA across this study group.

The remainder of the diastolic filling variables significantly correlated with BSA (or a transformation of BSA). Peak filling velocities, EA ratio, velocity integrals, and velocity
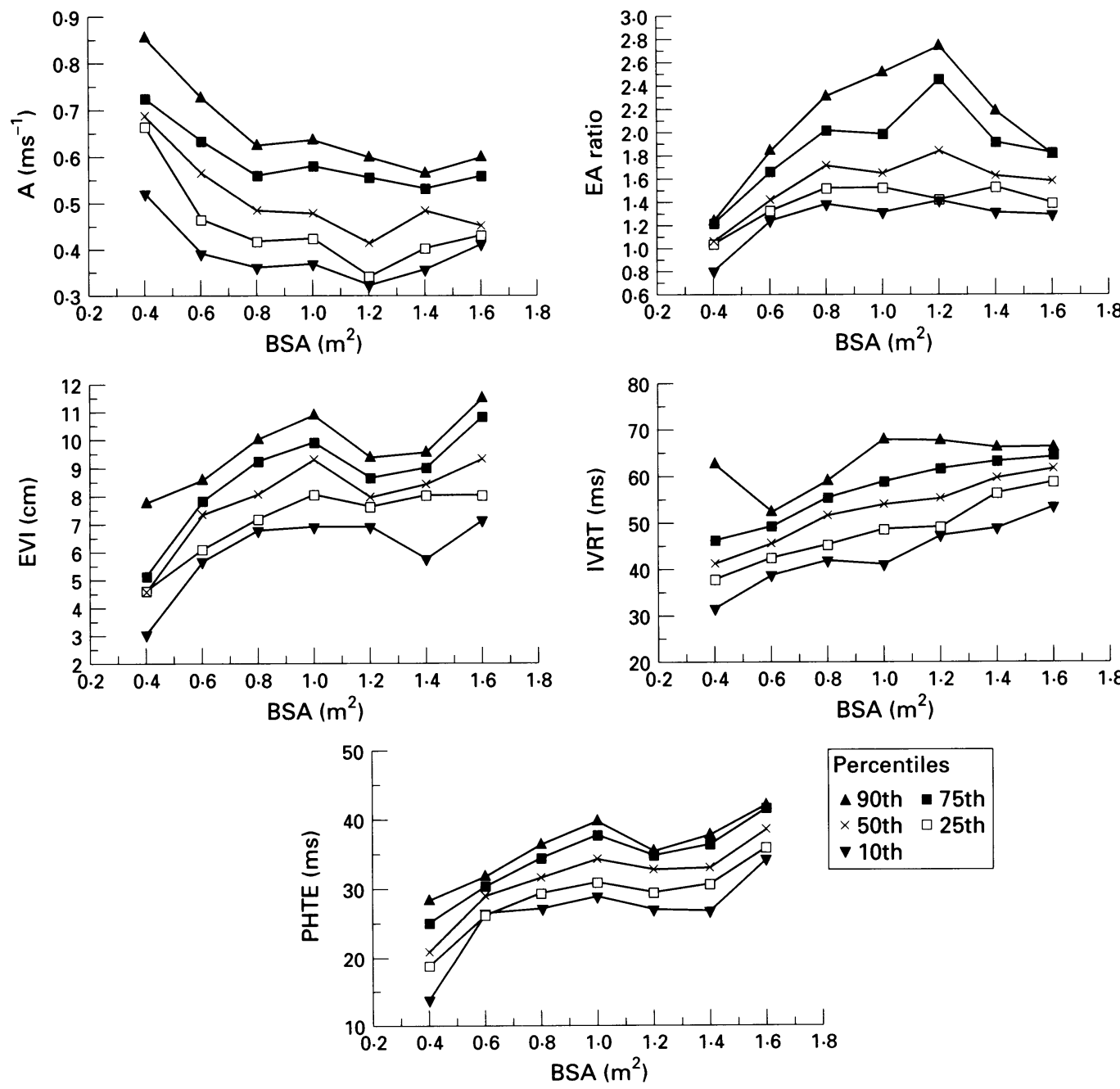

Figure 2 Centile charts for the most commonly used Doppler echocardiographic parameters of diastolic function in relation to body surface area (BSA) calculated using data from 130 normal participants. The 10th, 25th, 50th, 75th, and 90th centiles were calculated for children $<0.5 \mathrm{~m}^{2}>1.5 \mathrm{~m}^{2}$ and within $0.2 \mathrm{~m}^{2} B S A$ groups between these limits and plotted at the median $B S A$ value for each $B S A$ group. Additional plots for other parameters and the corresponding

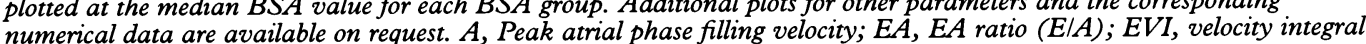
numerical data are available on request. $A$, Peak atrial phase filling velocity
of $E$ wave; IVRT, isovolumic relaxation time; PHTE, pressure half time. 
integral ratio all had a more linear relationship with heart rate than with BSA, but the relation remained weak for peak $E$ velocity and $A$ velocity integral. Although overall resting heart rate is inversely proportional to BSA in healthy children, there is significant variability and thus, range of normal, for a given BSA. Therefore the 10th, 25th, 50th, 75th, and 90th centile values for each variable were calculated for children with BSAs $<0.5 \mathrm{~m}^{2}$, $\geqslant 1.5 \mathrm{~m}^{2}$, and within $0.2 \mathrm{~m}^{2}$ BSA groups from 0.5 to $1.49 \mathrm{~m}^{2}$. Centile plots of the relation of the most frequently used diastolic variables with BSA are shown in fig 2. Plots for the other study variables, and the corresponding numeric data are available from the authors on request.

Cross correlations between transmitral flow variables were examined (table 1). The PHTE from peak $\mathrm{E}$ velocity mainly correlated with BSA $(r=0.67)$, in keeping with its relation with mitral valve area. IVRT also correlated with BSA $(r=0.56)$ and to a lesser degree to heart rate $(r=-0.47)$ and $\mathrm{E}$ deceleration $(r=-0 \cdot 5)$, but showed no noteworthy relation with any other variable.

There were no sex related differences detectable by comparative regression analysis in the relations between diastolic filling variables and BSA.

\section{Discussion}

Normal values for left ventricular diastolic filling variables change with age and this provides some interesting insights into left ventricular diastolic function in childhood. Although in healthy children, BSA and age are closely correlated, this is frequently not the case in sick children, including those with cardiac dysfunction. Normal data are therefore presented in relation to body size (BSA) rather than age.

The change in profile of the transmitral Doppler flow velocity envelope with growth is a result of the interdependence of filling variables (shown in table 1) and their relations with BSA and heart rate:

- Peak early filling velocity is most strongly related to the rate of acceleration to peak $E$, which depends principally on the atrioventricular pressure gradient. ${ }^{15}$ This is inversely related (among other factors) to mitral valve area and therefore also to BSA, but is largely unrelated to heart rate. Acceleration time is inversely proportional to heart rate and acceleration to peak $E$. Thus peak $E$ velocity is largely independent of BSA and only weakly inversely related to heart rate.

- The velocity integral of the $E$ wave is strongly correlated with deceleration time from peak E, which, like acceleration time, has similar but opposite relations with heart rate and BSA. As the heart rate decreases (with increased BSA), total early filling time lengthens. Therefore, despite little change in peak $E$ velocity, $E$ velocity integral and EA velocity integral increase with $B S A$ as heart rate decreases. These findings are in agreement with observations ${ }^{16}$ and pacing studies $^{17} 18$ in adults, although absolute relations differ. This might simply be the result of the much wider range of heart rates seen in the present participants. It might also reflect true physiological differences, however, between changes in diastolic function and alteration of heart rate in the same individual as opposed to cross sectional relations in the normal resting state.

- Peak atrial phase filling velocity is not related to the velocity integral of the $E$ wave but is principally related to heart rate in this study. Heart rate is known from studies in normal adults ${ }^{1718}$ to have a major effect on peak A velocity and therefore on EA ratio. This is probably the result of the shorter time available for early diastolic filling at faster heart rates, ${ }^{15}$ with a greater atrioventricular pressure gradient during atrial systole. The velocity integral of the $A$ wave is related to peak A velocity but shows little overall change with growth.

Thus, the lengthening of diastole at slower heart rates affects early and atrial phases of diastolic filling; the prolongation of early filling time is associated with a lower peak A velocity, but the constancy of the A velocity integral suggests that late filling also continues for longer. The increased total filling at higher BSAs (and lower heart rates) is therefore largely the consequence of an increase in the proportion of left ventricular filling occurring "passively" during early diastole.

The relation of IVRT to BSA, age, and deceleration from peak $\mathrm{E}$, with a weaker relation to heart rate, is largely as expected. With a larger ventricle and slower heart rate, the time taken during early diastolic relaxation for left ventricular pressure to decrease below left atrial pressure is increased and transmitral flow begins later. The negative correlation between IVRT and acceleration to peak E $(r>$ $-0.7)$ demonstrated in adults by Brecker et $a l^{19}$ was not found in these children. The adult group, however, had significant myocardial pathology with inco-ordinate relaxation, in addition to lower heart rates (the group mean heart rate was only 75 beats $\mathrm{min}^{-1}$ ). Such relations are clearly different in healthy children.

Normal values for PHTE in children have not previously been reported. This variable is clearly related to BSA in children, and, as in adults, may provide a guide to functional mitral valve area which might be of additional benefit in the evaluation of mitral stenosis.

These data provide new and potentially useful information on normal diastolic function in children. Concerns relating to the influence of loading conditions on results obtained as well as to technical factors in data acquisition, however, must also be addressed.

\section{LOADING CONDITIONS}

There is no doubt that transmitral diastolic flow patterns are affected by loading conditions. ${ }^{51520}$ By selecting 130 healthy, resting but unsedated children, the values obtained in this study incorporate normal variations in physiological loading conditions without 
pharmacological or physical manipulations, as reflected in the recorded measurements of blood pressure and heart rate. When making comparisons with these normal data, it is important to be aware that abnormal loading conditions are often associated with pathological processes and that either or both factors may influence the variables measured.

\section{RESPIRATORY PHASE}

It is well recognised that the respiratory phase may have an effect on transmitral flow patterns. ${ }^{21-23}$ There are considerable practical difficulties involved in obtaining detailed echocardiographic records from small children with ECG leads and a phonocardiogram attached. The use of a respiratory phase detector was considered to impose additional complications which could not be justified in healthy children. Thus there is no direct compensation for the respiratory phase in this study. It was felt, however, that selection of multiple similar Doppler complexes fulfilling certain predefined criteriathat is, those with the highest $\mathrm{E}$ wave and a clear A wave, was likely to minimise the variability due to the respiratory cycle. These are likely to be expiratory cycles as peak $\mathrm{E}$ velocity decreases during inspiration..$^{21-23}$

\section{SAMPLE VOLUME POSITION}

Studies in adults ${ }^{24}$ and infants ${ }^{25}$ have demonstrated differences in transmitral flow velocities obtained in the same individual at different locations within the left ventricular inflow tract. In common with many other groups, ${ }^{5-7}$ sample volume location was standardised at the tips of the mitral valve leaflets. This was because personal experience suggested greater reproducibility of sample volume siting at this location and also because it is suggested that the higher velocities obtained at this point best reflect the driving force across the mitral valve. ${ }^{5}$

\section{REPRODUCIBILITY}

Measurement error may arise at the time of scanning and analysis. The magnitude of interoperator and interanalyser variability has been demonstrated to be mainly between 5 and $10 \%$ in healthy adults. ${ }^{26}{ }^{27}$ Our own studies of reproducibility in 22 children with anthracycline cardiomyopathy yielded similar results, with only random variation for interoperator differences. These studies also demonstrated the potential for small but significant systematic variability in data analysis (for peak E velocity, A velocity integral, and deceleration time), although there was good correlation between analysers for all variables. Careful audit of data analysis results is required to ensure uniformity of measurement criteria if scans are to be analysed by more than one individual. The adult studies ${ }^{26}{ }^{27}$ also addressed within subject variability on different days (without controlling for operator) and noted similar findings; generally $5-10 \%$ variation, with more error for flow time measurements than for absolute velocities.
Although different sources of measurement error are not mutually exclusive, the magnitude of errors introduced by multiple operators, analysers, and examinations are likely to be cumulative. Thus, particularly for serial studies of individual patients, it is important to minimise other sources of error by reducing the number of operators and analysers, preferably to unity. Moreover, fewer effects of variability will occur with larger numbers of participants in such a series.

\section{Conclusions}

Standard normal data for healthy children may be used for comparative purposes in other studies. Such data are presented graphically here as centiles for particular BSA intervals. They portray actual observed data without statistical assumptions and provide a ready gauge of the degree of departure from the observed normal range for a given individual's function. These may be of particular value with serial studies of individual patients.

Transmitral diastolic flow patterns are strongly related to BSA. Some variables, however, show a degree of additional variability with resting heart rate. Differential effects of heart rate variation within the same individual were not examined here. Comparative studies of left ventricular function in children require BSA matching; the effects of heart rate in pathological situations also need careful consideration.

Doppler echocardiographic assessment of transmitral flow is relatively simple to perform, but the relations of the variables obtained to diastolic performance are less clear cut. The normal ranges for children (who are not small adults) are reported here, to permit further examination of these variables in pathological states. When applying these normal data to clinical or experimental results, the precise study protocols used and participants examined will have an important bearing on their accuracy and relevance. The relevance of these data in the structurally abnormal heart is not defined. The variability of transmitral flow velocity variables means that in clinical use, standardisation of echocardiographic examination and analysis techniques is vitally important. Perhaps guidelines similar to those of the American Heart Association for $M$ mode echocardiography ${ }^{28}$ are now indicated?

The authors thank Mrs M Shapland (Bristol University Computer Service) for database design and help with data handling, Mr J Halsey (Medical Research Council Clinical Trials Service Unit, Radcliffe Infirmary, Oxford) for statistical computing, and Mrs V Smith for data entry. FAB was sup ported by a grant from the Cancer Research Campaign and MGM is supported by the Cancer and Leukaemia in Childhood Trust.

1 Gutgesell HP. Echocardiographic assessment of cardiac function in infants and children. $f$ Am Coll Cardiol 1985 5:95-103s.

2 Cohn JN, Johnson G. Heart failure with normal ejection fraction: the V-HeFT study. Circulation 1990;81 (suppl III):48-53.

3 Grossman W. Diastolic dysfunction and congestive heart failure. Circulation 1990;81(suppl III):1-7. 
4 Nishamura RA, Housmans PR. Assessment of diastolic function of the heart: background and current applications of Doppler echocardiography: Part 1 . Physiologic and pathophysiologic features. Mayo Clin Proc 1989; 64:71-81.

5 Nishamura RA, Abel MD, Hatle LK, Tajik J. Assessment of diastolic function of the heart: background and current applications of Doppler echocardiography: Part II. applications of Doppler echocardiography: Part

6 Appleton CP, Hatle LK, Popp RL. Relation of transmitral flow velocity patterns to left ventricular diastolic function velocity patterns to left ventricular diastolic function: new insights from a combined hemodynamic and Doppler echocar

7 Spirito P, Maron BJ. Doppler echocardiography for assessing left ventricular diastolic function. Ann Intern Med 1988;109:122-6.

8 Johnson GL, Moffett CB, Noonan JA. Doppler echocardiographic studies of diastolic ventricular filling pattern in premature infants. Am Heart f 1988;116:1568-74.

9 Riggs TW, Rodriguez R, Snider AR, Batton DL. Doppler echocardiographic evaluation of right and left ventricular diastolic function in normal neonates. $尹 \mathrm{Am}$ Coll Cardiol 1989;13:700-5.

10 Loeber CP, Goldberg SJ, Allen HD. Doppler echocardiographic comparison of flows distal to the 4 cardiac valves. $₹$ Am Coll Cardiol 1984;4:268-72.

11 Labovitz AJ, Pearson AC. Evaluation of left ventricular diastolic function: clinical relevance and recent echocardiastolic function: clinical relevance and recent

12 Grenadier E, Oliveira C, Allen HD, et al. Normal intracardiac and great vessel Doppler flow velocities in infants and children. $₹ \mathrm{Am}$ Coll Cardiol 1984;4:343-50.

13 Kuo LC, Quinones MA, Rokey R, Sartori M, Abinader EG, Zoghbi WA. Quantification of atrial contribution to left ventricular filling by pulsed Doppler echocardiography and the effects of age in normal and diseased hearts. Am $\mathcal{f}$ Cardiol 1987;59:1174-8.

14 Smith SA, Stoner JE, Russell AE, Sheppard JM, Aylward PE. Transmitral velocities measured by pulsed Doppler in healthy volunteers: effects of acute changes in blood pressure and heart rate. Br Heart $\mathcal{1} 1989 ; 61: 344-7$.

15 Thomas JD, Weyman AE. Echocardiographic Doppler evaluation of left ventricular diastolic function: physics and physiology. Circulation 1991;84:977-90.

16 Stewart RAH, Joshi J, Alexander N, Nihoyannopoulos P, Oakley $C$. Adjustment for the influence of age and heart rate on Doppler measurements of left ventricular filling. rate on Doppler measureme
$B r$ Heart $\mathcal{F} 1992 ; 68: 608-12$.
17 Oniki T, Hashimoto Y, Shimizu S, Kakuta T, Yajima M, Numano F. Effect of increasing heart rate on Doppler indices of left ventricular performance in healthy men. Br Heart $\mathcal{f}$ 1992;68:425-9.

18 Harrison MR, Clifton GD, Pennell AT, DeMaria AN. Effect of heart rate on left ventricular diastolic transmitral flow velocity patterns assessed by Doppler echocardiography in normal subjects. Am $\mathcal{F}$ Cardiol 1991;67: 622-7.

19 Brecker SID, Lee CH, Gibson DG. Relation of left ventricular isovolumic relaxation time and incoordination to transmitral Doppler filling patterns. Br Heart $\mathcal{F}$ 1992;68: transmit

20 Stoddard MF, Pearson AC, Kern MJ, Ratcliff J, Mrosek DG, Labovitz AJ. Influence of alteration in preload on the pattern of left ventricular diastolic filling as assessed by Doppler echocardiography in humans. Circulation 1989;79:1226-36.

21 Dabestani A, Takenaka K, Allen B, et al. Effects of spontaneous respiration on diastolic left ventricular filling assessed by pulsed Doppler echocardiography. $A m \mathcal{F}$ Cardiol 1988;61:1356-8.

22 Riggs TW, Snider AR. Respiratory influence on right and left ventricular diastolic function in normal children. $A m$ f Cardiol 1989;63:858-61.

23 Uiterwaal C, Van Dam I, De Boo T, et al. The effect of respiration on diastolic blood flow velocities in the human heart. Eur Heart $\mathcal{f} 1989 ; 10: 108-12$.

24 Gardin JM, Dabestani A, Takenaka K, et al. Effects of imaging view and sample volume location in evaluation of mitral flow velocity by pulsed wave Doppler echocarof mitral flow velocity by pulsed wave Dopp

25 Johnson GL, Moffett CB, Jurnalov CD, Noonan JA. Effect of sample volume location on pulsed Doppler diastolic flow parameters in newborn infants. Pediatr Cardio 1988;9:221-4.

26 Fast J, van Dam I, Heringa A, et al. Limits of reproducibility of mitral pulsed Doppler spectra. Am $\mathcal{f}$ Cardiol 1988;61:891-4.

27 Spirito P, Maron BJ, Verter J, Merrill JS. Reproducibility of Doppler echocardiographic measurements of left ventricular diastolic function. Eur Heart $f$ 1988;9: 879-86.

28 Sahn DJ, De Maria A, Kisslo J, Weyman A. Recommendations regarding quantitation in $M$-mode echocic mittee on M-mode standardisation of the American Society of Echocardiography. Circulation 1978;58:1072-83. 